\title{
In vivo proliferation of differentiated pancreatic islet beta cells in transgenic mice expressing mutated cyclin-dependent kinase 4
}

\author{
S. Hino ${ }^{1,2}$ - T. Yamaoka ${ }^{1}$ Y. Yamashita ${ }^{1}$ T. Yamada Y J. Hata $^{3,4} \cdot$ M. Itakura ${ }^{1}$ \\ ${ }^{1}$ Division of Genetic Information, Institute for Genome Research, The University of Tokushima, Tokushima, Japan \\ 2 Department of Oral and Maxillofacial Surgery, School of Medicine, Ehime University, Ehime, Japan \\ ${ }^{3}$ Department of Pathology, School of Medicine, Keio University, Tokyo, Japan \\ ${ }^{4}$ National Center for Child Health and Development, Tokyo, Japan
}

\section{Abstract}

Aims/hypothesis. It has previously been hypothesised that highly differentiated endocrine cells do not proliferate or regenerate. However, recent studies have revealed that cyclin-dependent kinase 4 (CDK4) is necessary for the proliferation of pancreatic islet beta cells. The aim of this study was to determine whether activation of CDK4 can potentially be used as a radical treatment for diabetes without malignant transformation.

Methods. We generated transgenic mice expressing mutant CDK4 under the control of the insulin promoter to examine the effect of activated CDK4 overexpression in the postnatal development of pancreatic islets.

Results. In the transgenic mice, total CDK4 protein expression was increased by up to 5-fold, with a concomitant increase in CDK4 activity indicated by the detection of phosphorylated $\mathrm{Rb}$ protein in pancreatic islets. Histopathologically, many cells tested positive for proliferating cell nuclear antigen, and pancreatic islets displayed hyperplasia due to the extreme proliferation of beta cells containing a large number of insulin granules. Pancreatic islet alpha, delta and PP cells did not increase. Over an 18-month observation period, the transgenic mice did not develop insulinoma. Levels of expression of GLUT1 and $c$-myc were comparable to those in the littermates of the transgenic mice. GLUT2 expression was identified in the pancreatic islets of transgenic mice. No significant differences in telomerase activities were detected between transgenic mice and their littermates. Transgenic mice were superior to their littermates in terms of glucose tolerance and insulin secretion in response to the intraperitoneal injection of glucose, and hypoglycaemia was not observed.

Conclusions/interpretation. Activated CDK4 stimulates postnatal pancreatic beta cell proliferation, during which the highly differentiated phenotypes of pancreatic islet beta cells are preserved without malignant transformation.

Keywords CDK4 - Cell differentiation - Cell proliferation $\cdot$ Pancreatic islet beta cell $\cdot$ Transgenic mice
Received: 16 April 2004 / Accepted: 29 June 2004 Published online: 6 October 2004

(C) Springer-Verlag 2004

M. Itakura $(\bullet)$

Division of Genetic Information,

Institute for Genome Research,

The University of Tokushima,

3-18-15, Kuramoto-cho, Tokushima 770-8503, Japan

E-mail: itakura@genome.tokushima-u.ac.jp

Tel.: +81-88-6339454, Fax: +81-88-6339455

\section{Introduction}

The balance of growth-stimulatory and growth-inhibitory signals precisely controls proliferation, growth arrest and differentiation in mammalian cells. A series of kinase complexes govern the growth-stimulatory

Abbreviations: CDK, cyclin-dependent kinase - CDK4 R24C, Arg24 to Cys substituted CDK4 · DIG, digoxigenin ·

DTT, dithiothreitol - GST, glutathione $S$-transferase ·

$\mathrm{H} \& \mathrm{E}$, haematoxylin and eosin · ipGTT, intraperitoneal glucose tolerance test - MEF, mouse embryonic fibroblast . PCNA, proliferating cell nuclear antigen $\cdot \mathrm{PP}$, pancreatic polypeptide $\cdot \mathrm{Rb}$, retinoblastoma protein $\cdot \mathrm{Tg}$, transgenic 
signals. Each kinase complex contains a regulatory subunit (cyclin) and a catalytic subunit (cyclin-dependent kinase $[\mathrm{CDK}]$ ), and the level of expression of cyclin and the extent of phosphorylation of CDK control its enzymatic activity. In response to mitogenic signals, cyclin D/CDK4, cyclin D/CDK6 and cyclin E/CDK2 complexes phosphorylate retinoblastoma $(\mathrm{Rb})$ protein. Initially, Ser780 is phosphorylated by the cyclin D/CDK4 complex, which is required for the transition from $G_{1}$ to $S$ phase of the cell cycle $[1,2]$. The CDK inhibitors play a central role as growth-inhibitory signals that bind to the cyclin/CDK complexes and inhibit their catalytic activity, thus negatively regulating cell cycle progression. The CDK inhibitors are classified into two families based on functional and structural homologies. The INK4 family of inhibitors (p16 INK4a, p15 INK4b, $\mathrm{p} 18^{\mathrm{INK} 4 \mathrm{c}}$ and p19INK4d) specifically bind to CDK4 and CDK6 and prevent their interaction with D-type cyclins. The Cip/Kip family is composed of p21 Cip1/Waf1, p27 Kip1 and p57 Kip2 and has a broader inhibitory profile, blocking the activities of cyclin D-, E- and A-dependent kinases [3].

It has previously been hypothesised that, like nerve cells, highly differentiated endocrine cells do not proliferate or regenerate. Advances in developmental biology and molecular biological techniques such as cell culture, transgenic (Tg) and knock-out and knock-in mice have allowed the molecular mechanisms of pancreatic development to be gradually elucidated. Recent studies have revealed that islet cells can regenerate even in adult pancreas [4]. This finding raises the possibility that impaired pancreatic islets or a decrease in the number of islet cells in diabetic patients can be restored. The targeted homozygous disruption of CDK4 (CDK4-/-) in mice results in a dramatic reduction in the number of pancreatic beta cells and a diabetic phenotype, including hyperglycaemia, ketosis, glucosuria and polydipsia $[5,6]$. Furthermore, these mice are small in size due to an overall reduction in the size of major organs, and are infertile due to defective spermatogenesis and a reduced number of Leydig cells, or limited prolactin production [5, 6]. Mouse embryonic fibroblasts (MEFs) derived from $\mathrm{CDK}^{-/-}$mice proliferate in a similar manner to wildtype MEFs. However, MEFs from $\mathrm{CDK}_{4}^{-/-}$mice show delayed re-entry into the $\mathrm{S}$ phase of the cell cycle following serum stimulation $[5,6]$. Re-expression of endogenous CDK4 in beta cells by crossing $\mathrm{CDK}^{-/-}$mice with transgenic mice that express the Cre recombinase under the control of the rat insulin promoter restores cell proliferation and normoglycaemia [7]. These results indicate that CDK4 is indispensable for normal pancreatic development. Conversely, the CDK4R24C/R24C knock-in mice carrying the Arg24 to Cys substituted CDK4 (CDK4 $\mathrm{R} 24 \mathrm{C}$ ) display pancreatic islet hyperplasia due to the excessive proliferation of beta cells [6]. These mice develop spontaneous multiple tumours with almost complete penetrance and increased susceptibility to chemical carcinogen-induced tumourigenesis $[6,8]$. The most common neoplasias are endocrine tumours and haemangiosarcomas [8]. MEFs derived from CDK4 ${ }^{\mathrm{R} 24 \mathrm{C} / \mathrm{R} 24 \mathrm{C}}$ mice are immortal and are insensitive to contact inhibition of cell growth [8]. This mutation was first identified in patients with hereditary melanoma and causes CDK4 to lose its affinity for $\mathrm{p} 16^{\mathrm{INK} 4 \mathrm{a}}$ without affecting its ability to bind cyclin D and form a functional kinase complex $[9,10]$. In contrast, this mutation does not affect the interaction of CDK4 with p21 Cip1/Waf1 or p27 Kip1 [9].

Conventional diabetes therapies are partially effective, but they are not necessarily directed toward the fundamental aetiology of the disease. Regenerative medicine is expected to provide a greater degree of physiological control of blood glucose concentrations. However, there are concerns that these therapies could cause the malignant conversion of targeted cells. To investigate these possibilities, we generated transgenic mice expressing CDK4 $R 24 C$ under the regulation of the insulin promoter in pancreatic islets (CDK4 R24C-Tg mice). We examined whether CDK4 plays an important role in the fetal and postnatal development of pancreatic islets, and whether CDK4 can be used as a molecular target for the radical treatment of diabetes.

\section{Materials and methods}

Generation of transgenic mice. The construction of the expression vector including the 1.9-kb human insulin promoter used to generate transgenic mice has been described previously [11]. Human CDK4 R24C cDNA was provided by D. Beach (Howard Hughes Institute, Cold Spring Harbor, N.Y., USA) and was inserted into the cloning site flanking the exon-intron organisation and a polyadenylation signal of the rabbit $\beta$-globin gene. The BssHII-excised fragment of this vector excluding the plasmid-derived sequence was used as the transgene (Fig. 1a).

The transgene was micro-injected into male pronuclei of fertilised eggs obtained from super-ovulated BDF1 (C57BL/6 $\times$ DBA2 F1) female mice crossed with males of the same strain. Injected eggs were implanted into the oviducts of pseudopregnant female mice and allowed to develop [12]. DNA was extracted from tail snips of live offspring using the proteinase $\mathrm{K} / \mathrm{SDS}$ method [11]. The integration of the transgene into the mouse genome was detected by PCR between a sense primer in exon 1 of the human insulin promoter (5'-GCATCAGAAGAGGCCATCAA-3') and an antisense primer in exon 3 of the rabbit $\beta$-globin gene ( $5^{\prime}$-ACTCACCCTGAAGTTCTCAG-3') and by Southern blot analysis. The SalI-NotI fragment of the transgene was used as a probe and compared with indicator bands of 1, 10 and 100 copies of the transgene. The F1 transgenic progeny were bred by crossing transgenic founder mice with BDF1 mice, and were used to observe phenotypes over the long term and to repeat crossing with BDF1 mice. The F2 transgenic mice and their sex-matched non-transgenic littermates (wild-type mice) were used in this study. The F2 transgenic mice were back-crossed to C57BL6 mice or DBA2 mice for four genera- 


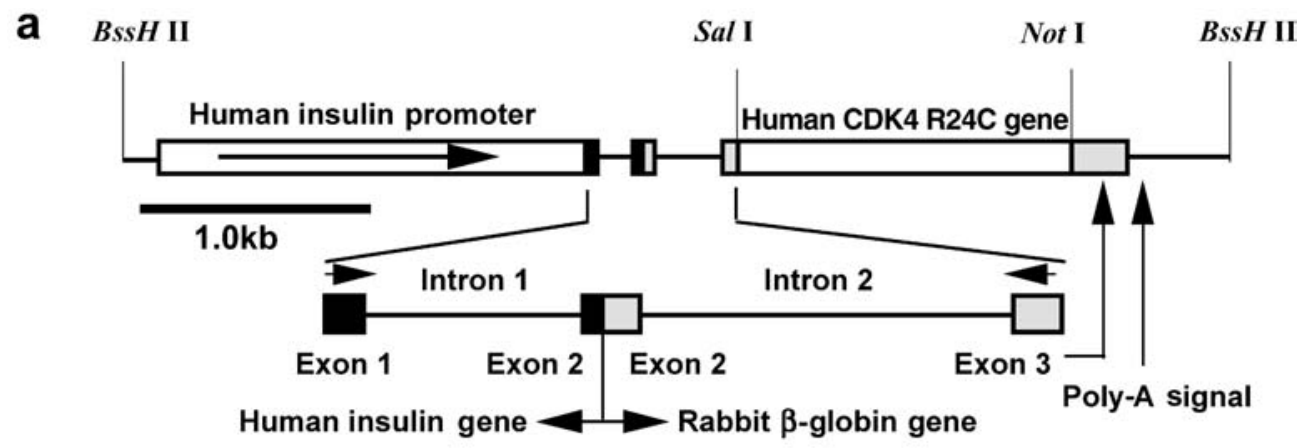

b

\begin{tabular}{|c|c|c|c|c|}
\hline \multirow[b]{2}{*}{$\mathbf{P}$} & \multirow[b]{2}{*}{$\mathbf{N}$} & \multicolumn{3}{|c|}{ Line number } \\
\hline & & 1 & 2 & 3 \\
\hline & & $=$ & $=$ & \\
\hline
\end{tabular}

C

\begin{tabular}{|c|c|c|c|c|c|}
\hline & d & & $\mathrm{Cc}$ & nu & ber \\
\hline 1 & 2 & 3 & 1 & 10 & 100 \\
\hline
\end{tabular}

d

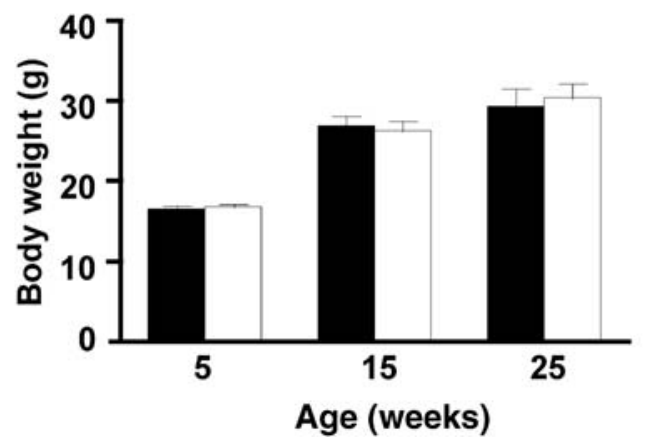

Fig. 1. Transgene construct and expression. a. The transgene construct used in this study. Human CDK4 R24C transgene was expressed in mouse pancreatic islets under the control of the human insulin promoter. b. In order to detect transgene-derived mRNA, a sense primer in exon 1 of the human insulin promoter (left horizontal arrow) and an antisense primer in exon 3 of the rabbit $\beta$-globin gene (right horizontal arrow) were synthesised for RT-PCR. P, positive control; N, negative control. c. The copy numbers of integrated transgene in lines 1 to 3 of the transgenic mice were examined by Southern blotting. d. Bar graph of the body weights of the mice at 5, 15 and 25 weeks of age. The results shown are the means \pm SEM of values for at least 13 mice. Black bars, littermates; white bars, CDK4 R24C-Tg mice tions. Body weight was determined weekly. All mice were supplied by CLEA Japan (Tokyo, Japan) and handled according to the "Principles of Laboratory Animal Care" (NIH Publication No. 85-23, revised 1985).

Transgene expression in pancreatic islets. Pancreatic islets were collected by the collagenase method [13, 14], total RNA was extracted with ISOGEN (Wako Pure Chemical Industries, Osaka, Japan) and reverse-transcribed by SuperScript Firststrand Synthesis System (Invitrogen, Carlsbad, Calif., USA) in $20 \mu \mathrm{l}$ of the reaction mixture. A $1-\mu \mathrm{l}$ aliquot of the products was subjected to PCR amplification. To determine whether conditions were adequate for semi-quantitative RT-PCR, we reverse-transcribed $0.01,0.1,1$ and $5 \mu \mathrm{g}$ of total RNA and amplified the fragments under the same PCR conditions using 15 to 30 cycles. We used $1 \mu \mathrm{g}$ of total RNA as a template for 
reverse transcription, and the cDNA fragment was amplified by using sense and antisense primers specific to human $C D K 4$ (5'-TACCTCTCGATATGAGCCAG-3' and 5'-CACCAGGGTTACCTTGATCT- $3^{\prime}$ ). Sense and antisense primers to $\beta$-actin were used as an internal standard (5'-GTGGGCCGCTCTAGGCACCA-3' and 5'-CGGTTGGCCTTAGGGTTCAGG-3'). After confirming the linear increase in the PCR product with increasing amounts of total RNA, we chose the middle cycle number of 15 to 30 cycles in the phase of linear logarithmic increment for each gene to semi-quantitate the mRNA expression in pancreatic islets. PCR products were separated by polyacrylamide gel electrophoresis and visualised with ethidium bromide. The expression of amylin (5'-TGTGCATCTCCAAACTGCCA-3' and 5'-GATTCCCTATTTGGATCCCC-3'), Pax4 (5'-ACACCAGGCAGCAGATTGTG-3' and 5'-CCTGTGCGGTAGTAGCGTCC-3'), $c$ - myc (5'-CGTGAACTTCACCAACAGGA-3' and $5^{\prime}$-CTCTGCTGTTGCTGGTGATA-3') and GLUT1 (5'-GAATCGTCGTTGGCATCCTT-3' and 5'-AGATGACACTGAGCAGCAGA-3') was examined similarly.

Immunoprecipitation, immunoblotting and the CDK4 kinase assay. Pancreatic islets collected by the collagenase method were washed three times with ice-cold PBS and lysed in lysis buffer containing $50 \mathrm{mmol} / \mathrm{l}$ HEPES (pH 7.5), 0.1\% Tween 20, $150 \mathrm{mmol} / \mathrm{l} \mathrm{NaCl}, 2.5 \mathrm{mmol} / \mathrm{l} \mathrm{EGTA}, 1 \mathrm{mmol} / \mathrm{l}$ dithiothreitol (DTT), 10\% glycerol, complete protease inhibitor cocktail (Roche, Mannheim, Germany), $10 \mathrm{mmol} / 1 \beta$-glycerophosphate, $1 \mathrm{mmol} / \mathrm{l} \mathrm{NaF}$, and $0.1 \mathrm{mmol} / \mathrm{l}$ sodium orthovanadate (all phosphatase inhibitors were from Sigma, St. Louis, Mo., USA). Sonicated extracts were clarified by centrifugation and the supernatants were used as whole cell lysates [15]. The protein concentrations of the supernatants were determined by the Bradford method (Bio-Rad, Hercules, Calif., USA). The supernatants were precipitated at $4{ }^{\circ} \mathrm{C}$ for $6 \mathrm{~h}$ with the indicated antibody, and immunoprecipitates were isolated using protein G-agarose beads (Roche) at $4{ }^{\circ} \mathrm{C}$ for $1 \mathrm{~h}$. The beads were subsequently washed four times with lysis buffer. For immunoblotting, boiling in SDS-PAGE sample buffer denatured whole cell lysates or immunoprecipitated beads. Denatured proteins in the supernatants were separated by SDS-PAGE and transferred onto Immobilon-P membranes (Millipore, Bedford, Mass., USA). Membranes were probed with antibodies to CDK4, Rb, $\beta$-tubulin (Pharmingen, San Diego, Calif., USA) or Rb phosphorylated at Ser780 (Cell Signaling Technology, Beverly, Mass., USA). Horseradish peroxidase-conjugated secondary antibody (Amersham Pharmacia Biotech, Little Chalfont, UK) and the Enhanced Chemiluminescence Detection System (Amersham Pharmacia Biotech) were used for signal detection.

The assay used to assess CDK4 kinase activity was as described previously [16]. In brief, glutathione $S$-transferase (GST)-tagged Rb proteins derived from pGEX.3X.mRb.C'-ter vector (a kind gift by Dr. H. Matsushime, Yamanouchi Pharmaceutical Company, Tokyo, Japan) were expressed in Escherichia coli BL21 (pLysS) and affinity purified using glutathione-Sepharose CL-4B (Amersham Pharmacia Biotech), according to the manufacturer's instructions. Whole cell lysate was incubated with an antibody to $\mathrm{CDK} 4$ at $4{ }^{\circ} \mathrm{C}$ for $6 \mathrm{~h}$. Antigen-antibody complexes were precipitated with protein G-agarose beads at $4{ }^{\circ} \mathrm{C}$ for $1 \mathrm{~h}$, which were then washed four times with lysis buffer. Recovered CDK4-cyclin D complexes were incubated at $30{ }^{\circ} \mathrm{C}$ for $30 \mathrm{~min}$ in $20 \mu \mathrm{l}$ of kinase buffer $\left(50 \mathrm{mmol} / \mathrm{l}\right.$ HEPES [pH 8.0], $10 \mathrm{mmol} / \mathrm{l} \mathrm{MgCl}_{2}$, $1 \mathrm{mmol} / \mathrm{l}$ DTT, $2.5 \mathrm{mmol} / \mathrm{l}$ EGTA, $10 \mathrm{mmol} / \mathrm{l} \beta$-glycerophosphate, $1 \mathrm{mmol} / 1 \mathrm{NaF}, 0.1 \mathrm{mmol} / \mathrm{l}$ sodium orthovanadate) supplemented with $20 \mu \mathrm{mol} / \mathrm{l}$ ATP, $3.7 \times 10^{5} \mathrm{~Bq}$ of $\left[\gamma^{32} \mathrm{P}\right]$ ATP (Amersham Pharmacia Biotech) and $0.5 \mu \mathrm{g}$ of purified GST-Rb substrate. Reactions were stopped by boiling in sample buffer and the denatured proteins were separated by electrophoresis through a $10 \%$ polyacrylamide gel. Phosphorylated proteins were visualised by autoradiography.

Examination of proliferation and differentiation markers of pancreatic islets. For the histopathological examination, mouse pancreas was fixed with $20 \%$ formalin in PBS, embedded in paraffin, sectioned and stained with haematoxylin and eosin (H\&E). For immunohistochemistry, guinea pig polyclonal antibodies for porcine insulin, glucagon, somatostatin and pancreatic polypeptide were used (DAKO Japan, Kyoto, Japan). Tumourigenesis of pancreatic islets was examined by insulin content, expression of GLUT1, GLUT2, Pax4,c-myc and amylin, with an SV-40-transformed pancreatic islet beta cell line of MIN6 used as a transformed insulinoma cell line. Fasting insulin concentration was used as an indicator of insulinoma. Mouse monoclonal antibodies were used to identify proliferating cell nuclear antigen (PCNA) (DAKO Japan) in proliferating cells and GLUT 2 in well-differentiated beta cells (Chemicon International, Temecula, Calif., USA). Quantitative analysis of the islet cell area was carried out as previously described [11].

Glucose tolerance test, HbAlc measurement, plasma insulin concentrations and insulin content in the whole pancreas. After an overnight fast, the intraperitoneal glucose tolerance test (ipGTT) was performed by intraperitoneally injecting $2 \mathrm{mg} / \mathrm{g}$ body weight of glucose in physiological saline. At 0, 30, 60 and $120 \mathrm{~min}$ after the injection, blood glucose concentrations were determined by the glucose oxidase method with a Diasensor (Kyoto Daiichi Kagaku, Kyoto, Japan). We measured $\mathrm{HbA}_{1} \mathrm{c}$ by ion exchange chromatography (Nippon Chemiphar, Tokyo, Japan) according to the manufacturer's protocol. Plasma insulin concentrations were assayed using an insulin ELISA kit with a mouse insulin standard (Seikagaku Kogyo, Tokyo, Japan). To determine insulin content in the whole pancreas, each pancreas was homogenised in $4 \mathrm{ml}$ of ice-cold acid-ethanol solution and insulin was extracted overnight at $4{ }^{\circ} \mathrm{C}$. After centrifugation, the supernatant was neutralised and diluted with PBS. Insulin concentrations were assayed in samples diluted 1000- or 10,000-fold [11, 17].

Determination of telomerase activities in pancreatic islets. Telomerase activities in pancreatic islets were measured using a telomerase PCR ELISA kit (Roche). Pancreatic islets were lysed in ice-cold lysis buffer for $30 \mathrm{~min}$, and $10 \mu \mathrm{g}$ of cell extract was analysed using a modified telomeric repeat amplification protocol (TRAP) [18]. Briefly, telomeric repeats are added to a biotin-labelled primer during the first reaction, then elongation products are amplified by PCR. An aliquot of the PCR product is denatured, hybridised to a digoxigenin (DIG)labeled telomeric repeat-specific probe and bound to a streptavidin-coated 96-well plate. The immobilised PCR product is detected with an anti-DIG-POD (peroxidase) antibody, visualised by a coloured reaction product generated using the substrate tetramethylbenzidine (TMB), and semiquantified photometrically at $\mathrm{A}_{450 \mathrm{~nm}} / \mathrm{A}_{690 \mathrm{~nm}}$.

Statistical analysis. All data are presented as means \pm SEM. For comparison of two means, the Student's unpaired $t$ test was used. For comparison of two ratios $\left(Q_{1}, Q_{2}\right)$, a normal distribution curve with a mean of $Q_{1}-Q_{2}$ and a variance of $Q_{1}\left(1-Q_{1}\right) / n_{1}+Q_{2}\left(1-Q_{2}\right) / n_{2}$ was used. A $p$ value less than 0.05 was considered statistically significant. 


\section{Results}

Generation of CDK4 R24C transgenic mice. The transgene construct used in this study is shown in Figure 1a. Three CDK4 R24C-Tg founder mice were obtained (Fig. 1b). The transgene copy numbers of CDK4 R24C-Tg mice in lines 1 to 3 were 30, 70 and 30 respectively (Fig. 1c). The percentages of F2 transgenic mice at 5 weeks of age were $56 \%$ (63 of 112) in line 1,54\% (41 of 76) in line 2 and 47\% (30 in 66) in line 3, which is proportional to Mendel's law. Body weights at the age of 5, 15 and 25 weeks are shown in Figure 1d; there were no significant differences between CDK4 R24C-Tg mice and their littermates. These results indicate that overexpressed CDK4 R24C does not affect embryogenesis and survival of the mice.

The expression of the transgene in the pancreatic islets of transgenic mice was confirmed by RT-PCR. Its expression was detected in lines 1 to 3 of CDK4 R24C-Tg mice, but was not detected in their littermates (Fig. 2a). The levels of expression of the transgene in the three lines were not always correlated with their copy numbers (Figs. 1c, 2a).

The expression of CDK4 R24C and endogenous CDK4 was examined by immunoblotting with antiCDK4 antibody. Levels of total CDK4 expression in the pancreatic islets of CDK4 R24C-Tg mice were up to five times higher than in their littermates (Fig. 2b). Thus, the level of expression of CDK4 R24C driven by the human insulin promoter was approximately four times higher than that of the endogenous wildtype CDK4 (Fig. 2b). CDK4 kinase activity against recombinant GST-Rb protein substrate was clearly detected in the pancreatic islets of CDK4 R24C-Tg mice, but not in those of their littermates (Fig. 2c). We also examined the phosphorylation status of $\mathrm{Rb}$ by immunoblotting with antibody directed against Ser780-phosphorylated $\mathrm{Rb}$. Levels of $\mathrm{Rb}$ expression were almost the same in the two groups of mice, but phosphorylation at Ser780 was only detected in the pancreatic islets of CDK4 R24C-Tg mice (Fig. 2d). These results suggest that the mitogenic signal is not transduced in normal pancreatic islets even in the presence of a sufficient supply of oxygen and nutrients.

Hyperplasia of pancreatic islets in CDK4 R24C transgenic mice. Haematoxylin and eosin staining of pancreases from 25-week-old mice revealed that islet area was increased in CDK4 R24C-Tg mice relative to that in their littermates (Figs. 3a, b). Islet area expressed as a percentage of the area of the whole pancreas was 10.2 times higher in the CDK4 R24C-Tg mice than in their littermates (Fig. 3e). Immunohistochemical analysis for insulin revealed that the enlargement of islet area in CDK4 R24C-Tg mice was due to the proliferation of beta cells (Figs. 3c, d). Beta cell area expressed
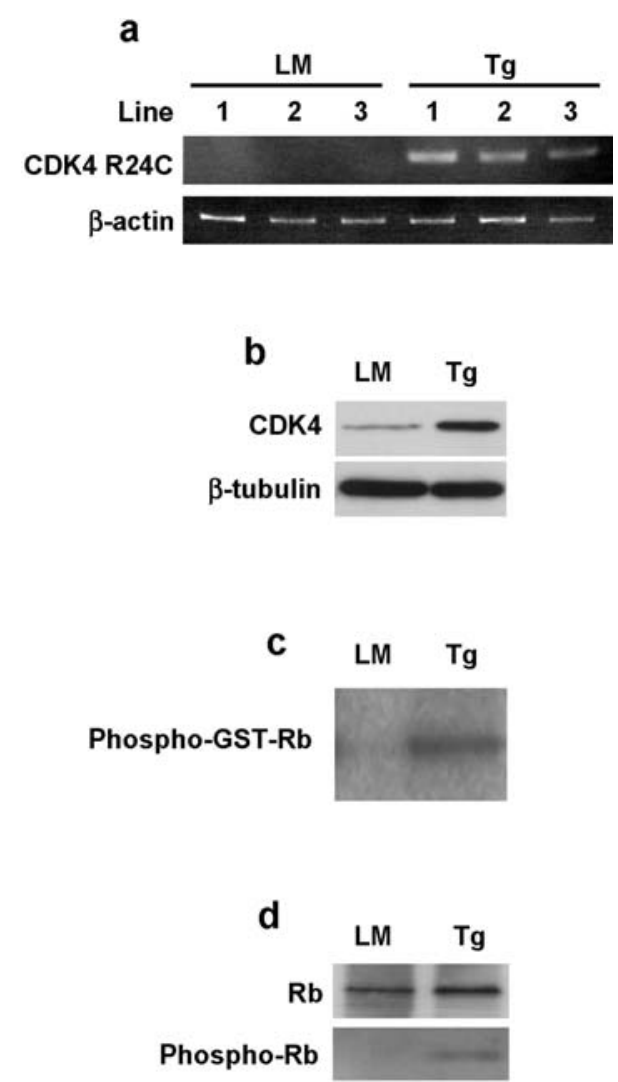

Fig. 2. CDK4 expression and its activity in CDK4 R24C-Tg mice islets. a. Total RNA was extracted from pancreatic islets (collected by the collagenase method) and applied to RT-PCR. In all lines of transgenic mice, RT-PCR products were amplified. b. Whole cell lysates were prepared from pancreatic islets as above and subjected to immunoblotting with anti-CDK4 antibody. This antibody recognises both wild-type CDK4 and CDK4 R24C. Expression of $\beta$-tubulin was measured as an internal control. c. CDK4 activity in pancreatic islets assayed using GST-Rb as a substrate after immunoprecipitation of CDK4. d. Antibody to $\mathrm{Rb}$ was used for immunoprecipitation, and precipitated $\mathrm{Rb}$ was analysed by immunoblotting with antibody specific for Ser780-phosphorylated Rb. After removing prior antibody, the total amount of precipitated $\mathrm{Rb}$ was determined by immunoblotting with antibody to Rb. LM, littermates; Tg, CDK4 R24C-Tg mice

as a percentage of the area of the whole pancreas was 14.3 times higher in CDK4 R24C-Tg mice than in their littermates (Fig. 3f). The areas of other pancreatic hormonal cells, including alpha, delta and pancreatic polypeptide (PP) cells, relative to the area of the whole pancreas were similar in the two groups of mice (Figs. 4a-m).

In order to define the initiation of beta cell proliferation in CDK4 R24C-Tg mice, we examined the islet area in neonatal mice (Day 0) and 5-week-old mice by immunohistochemical staining for insulin. At 5 weeks, islet area was increased in CDK4 R24C-Tg mice due to the proliferation of beta cells, whereas no significant differences were observed between CDK4 R24C-Tg mice and their littermates at Day 0 (Figs. 5a-f). At 5 weeks, islet area expressed as a 

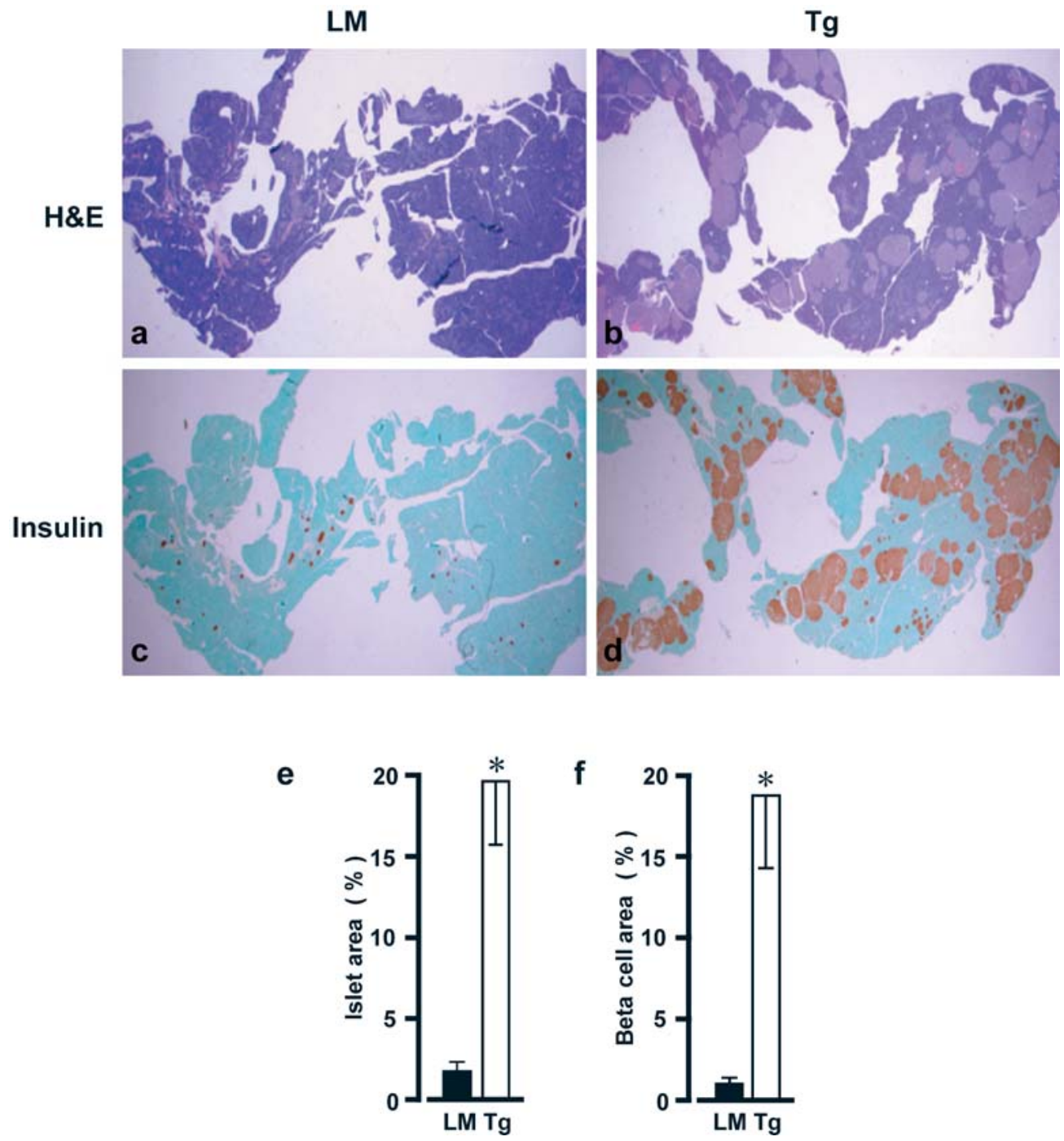

Fig. 3. Histopathological examination of pancreatic islets. At 25 weeks, sections of pancreas from littermates (a, c) and CDK4 R24C-Tg mice $(\mathbf{b}, \mathbf{d})$ were evaluated by H\&E staining (a, b) and anti-insulin immunohistochemistry (c, d). Islet area (e) and beta cell area (f) relative to the area of whole pancreas ( $n=7$ each). Both areas were significantly increased in CDK4 R24C-Tg mice (* $p<0.01$ vs LM). LM, littermates; Tg, CDK4 $\mathrm{R} 24 \mathrm{C}-\mathrm{Tg}$ mice

percentage of the area of the whole pancreas was 3.9 times higher in CDK4 R24C-Tg mice than in their littermates (Fig. 5f). There were no significant differences in the areas of other pancreatic islet endocrine cells between CDK4 R24C-Tg mice and their littermates at 5 weeks and 25 weeks (data not shown).

Glucose tolerance. In spite of the remarkable enlargement of pancreatic islets, hypoglycaemia was not observed in CDK4 R24C-Tg mice. Levels of $\mathrm{HbA}_{1} \mathrm{c}$ in CDK4 R24C-Tg mice $(1.22 \pm 0.06 \%, n=30)$ were similar to those in their littermates $(1.29 \pm 0.06 \%, n=31)$. Blood glucose concentrations examined at $0,30,60$ and $120 \mathrm{~min}$ after glucose injection at 5, 15 and 25

weeks of age were significantly lower in CDK4 R24C-Tg mice than in their littermates at all time points (Figs. 6a-c). Because body weight increases with age, the glucose tolerance of the littermates worsened, as indicated by the sustained high glucose levels at 60 and $120 \mathrm{~min}$ after glucose injection in the 15- and 25-week-old mice (Figs. 6b, c). In contrast, the glucose tolerance of the CDK4 R24C-Tg mice did not alter with age.

Insulin secretion and storage. Plasma insulin concentrations were determined at $0,30,60$ and $120 \mathrm{~min}$ after glucose injection. Insulin secretion in CDK4 R24C-Tg mice at 25 weeks of age was statistically higher than that in their littermates at all time points (Fig. 6d). The insulin content of pancreases from CDK4 R24C-Tg mice $(108 \pm 30 \mu \mathrm{g} /$ pancreas g) was 2.8 times higher than that in the pancreases of their littermates $(39 \pm 7 \mu \mathrm{g} /$ pancreas g) $(p<0.05, n=10$ in both groups). The whole pancreatic weight was similar in both mice (data not shown).

Proliferation and differentiation of pancreatic islet beta cells. The proliferation of beta cells was con- 

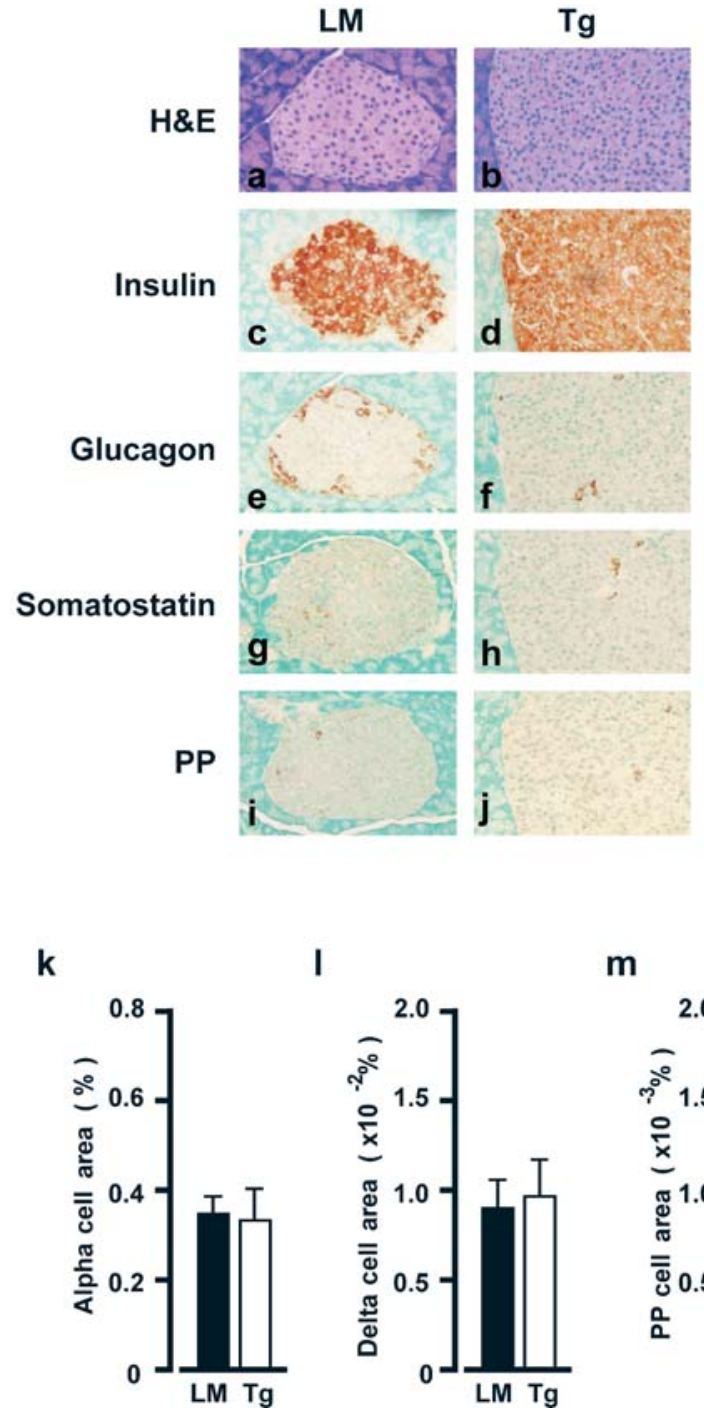

I

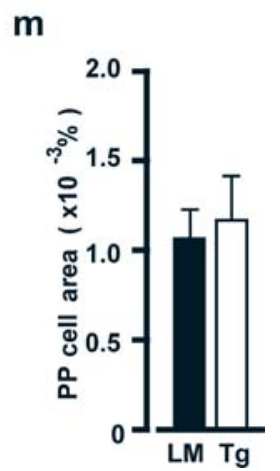

Fig. 4. Immunohistochemical analysis of pancreatic islets. Pancreata of littermates (a, c, e, g, i) and CDK4 R24C-Tg mice $(\mathbf{b}, \mathbf{d}, \mathbf{f}, \mathbf{h}, \mathbf{j})$ at 25 weeks of age were examined by H\&E staining $(\mathbf{a}, \mathbf{b})$ and immunohistochemistry against insulin (c, d), glucagon $(\mathbf{e}, \mathbf{f})$, somatostatin $(\mathbf{g}, \mathbf{h})$ and PP $(\mathbf{i}, \mathbf{j})$. The areas of alpha, delta and PP cells $(\mathbf{k}, \mathbf{l}, \mathbf{m})$ relative to the area of the whole pancreas were similar in both groups of mice $(n=7$ each). LM, littermates; Tg, CDK4 R24C transgenic mice

firmed by positive staining against PCNA in CDK4 R24C-Tg mice (Fig. 7b). Conversely, hardly any proliferating beta cells were detected in their littermates (Fig. 7a). In order to investigate the degree of beta cell differentiation in CDK4 R24C-Tg mice, the expression of differentiation marker molecules for pancreatic islet beta cells was examined by RT-PCR and immunohistochemical analysis. Pancreatic islet beta cells in CDK4 R24C-Tg mice contained abundant insulin granules and immunohistochemical analysis for insulin showed them to be strongly stained (Figs. 3d, 4d). The level of expression of GLUT2, a marker of highly differentiated beta cells, was similar in the two groups of mice (Figs. 7c, d). Pax4 is expressed only in poorly differentiated beta cells, GLUT1 is specifically expressed in beta cells transformed into insulinoma cells, and the expression of c-myc is increased in beta cells transformed into insulinoma cells. In the present study, this pattern of expression was observed in an insulinoma cell line of MIN6 cells, but not in the pancreatic islets of CDK4 R24C-Tg mice or their littermates (Fig. 7e). The results showed that amylin is expressed in pancreatic islet beta cells regardless of their degree of differentiation (Fig. 7e). Moreover, insulinoma did not develop in CDK4 R24C-Tg mice over an 18-month observation period, as confirmed by histopathological analysis (data not shown).

Determination of telomerase activities. As shown in Figure 8, telomerase activities increased in the pancreatic islets of both groups of mice in an age-dependent manner. Telomerase activities in the pancreatic islets of CDK4 R24C-Tg mice were higher than in those of their littermates, but no significant differences were detected. These results suggest that the pancreatic islets did not acquire the malignant phenotype by CDK4 $\mathrm{R} 24 \mathrm{C}$ overexpression.

Effect of genetic background on islet area and glucose tolerance. Islet area in the $\mathrm{N} 4$ generation of CDK4 R24C-Tg mice, back-crossed to C57BL/6 and DBA2 mice, was significantly increased in both strains in comparison with their littermates ( $p<0.05$ for both). In their littermates, the islet area in C57BL/6 mice was significantly smaller than in DBA2 mice $(p<0.01)$. In both strains, glucose concentrations measured at different time points during the ipGTT were significantly lower in the CDK4 R24C-Tg mice than in their littermates $(p<0.05$ for both). Among the littermates, glucose concentrations were significantly lower in DBA2 mice than in $\mathrm{C} 57 \mathrm{BL} / 6$ mice $(p<0.01)$.

\section{Discussion}

In three lines of CDK4 R24C-Tg mice, we observed a high number of copies of the transgene and generation rates of transgenic F2 mice according to Mendel's law. In other words, we have demonstrated that the expression of CDK4 R24C in pancreatic beta cells does not produce any concomitant changes in the development and growth of mice.

The over-proliferation of pancreatic islet beta cells was observed in 5-week-old CDK4 R24C-Tg mice, but not in neonatal CDK4 R24C-Tg mice. Even at 25 weeks, the beta cells of CDK4 R24C-Tg mice expressed PCNA protein, indicating that the proliferation of beta cells continues at least up to 25 weeks of age. The lack of proliferation of the beta cells of the neonatal CDK4 R24C-Tg mice may be explained by insufficient insulin promoter activity of the fetus. The pancreatic islets in fetal mice do not produce or 

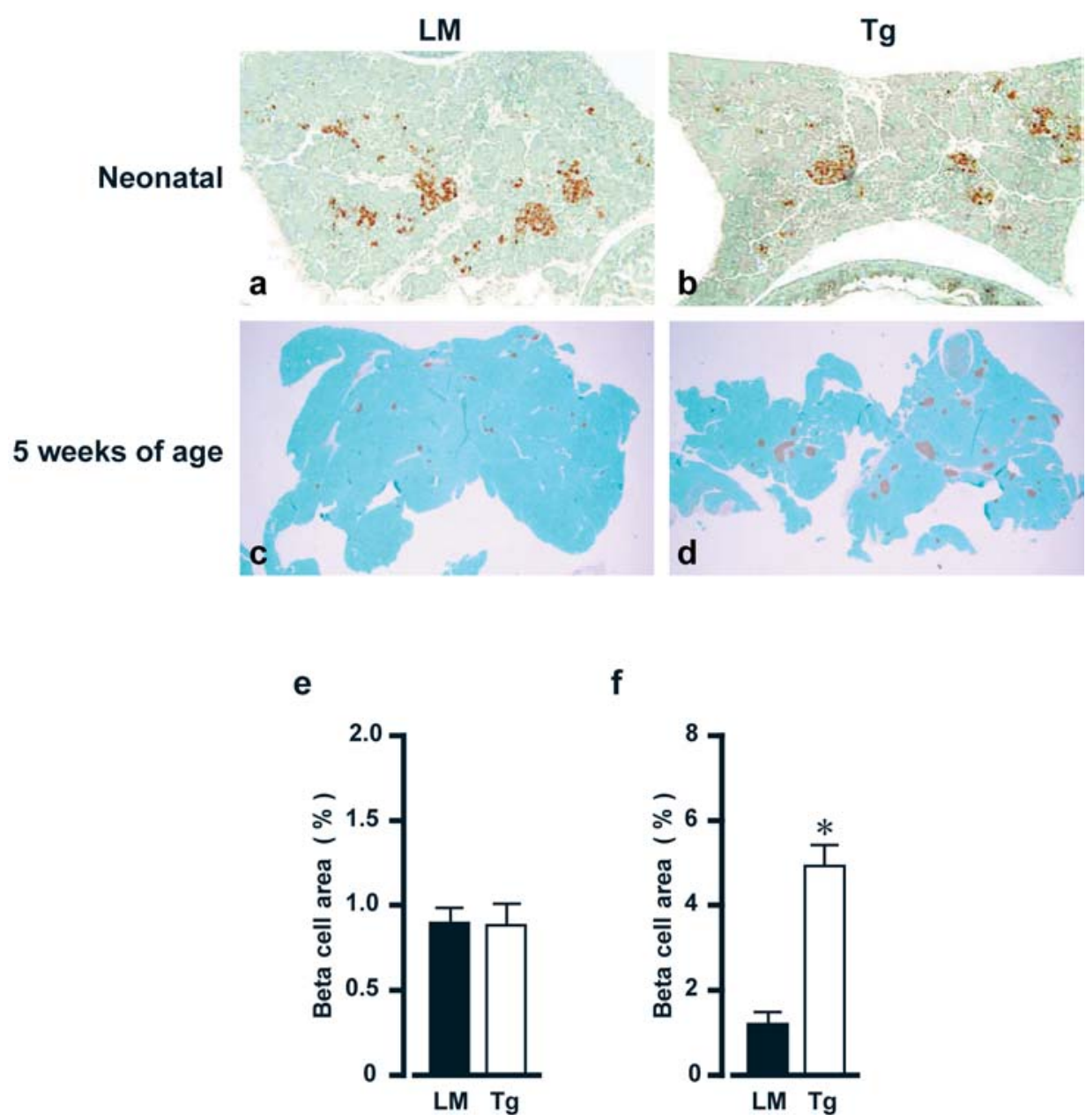

f

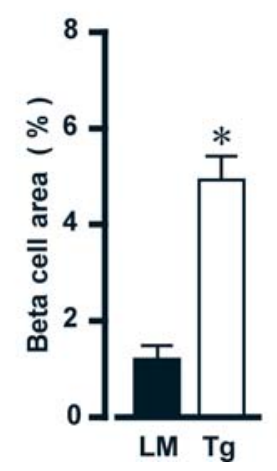

Fig. 5. Immunohistochemical analysis of pancreatic islets in neonatal mice and 5-week-old mice. Pancreata from littermates (LM) (a, c) and CDK4 R24C-Tg (Tg) (b, d) at neonatal Day 0 (a, b) and 5 weeks (c, d) were examined by immunohistochemistry for insulin. The beta cell area relative to the whole area of pancreas was determined ( $n=7$ each). The beta cell areas were similar in the two groups of neonatal Day 0 mice (e), but were significantly increased in CDK4 R24C-Tg mice at 5 weeks of age (f) $(* p<0.05$ vs littermates)

secrete insulin in response to glucose stimulation, and blood glucose concentrations in fetal mice are mainly maintained by maternal insulin [19, 20, 21, 22]. Under the limited activity of the insulin promoter in fetal pancreatic islets, CDK4 R24C expression was not high enough to proliferate beta cells in fetal mice.

After birth, the differentiation and maturation of beta cells are induced by the demand for them to secrete insulin; thus, activation of the insulin promoter would be required to increase the expression of the CDK4 R24C transgene in order to induce the proliferation of beta cells. Beta cell areas in $\mathrm{CDK}^{-/}$and CDK4 ${ }^{\mathrm{R} 24 \mathrm{C} / \mathrm{R} 24 \mathrm{C}}$ mice at Day 15.5 of gestation and Day 5 of age have been demonstrated to be comparable to those in their littermates [7]. Furthermore, the nuclear translocation of CDK4 is developmentally regulated

to coincide with the initiation of beta cell proliferation. Based on these results, another possibility is that CDK4 itself is not essential for beta cell neogenesis in the mouse embryo.

Over an 18-month observation period, insulinoma did not develop in CDK4 R24C-Tg mice as confirmed by histopathological examination. Hyperplasia of pancreatic islet beta cells was diagnosed in CDK4 $\mathrm{R} 24 \mathrm{C}-\mathrm{Tg}$ mice based on the following findings. Proliferation of beta cells occurred with the concomitant polyclonal enlargement of many islets. The ratio of nuclear : cytoplasmic area did not increase in the CDK4 R24C-Tg mice. The size and number of nucleoli were also similar in the two groups. In addition to the proliferating beta cells, a comparable number of alpha, delta, and PP cells were detected in pancreatic islets of CDK4 R24C-Tg mice and their littermates. Furthermore, the expression of GLUT2 (a molecular marker of highly differentiated beta cells) [23], the lack of expression of Pax4 [24] and GLUT1 [23] and the lack of an increase in $c$-myc expression $[25,26]$ indicated the absence of insulinoma development. No significant increase in telomerase activity was detected as a result of the overexpression of CDK4 R24C. In a previous study, the expression of CDK4 R24C alone had little effect on the life span of primary human keratinocytes, but simultaneous expression of 
a
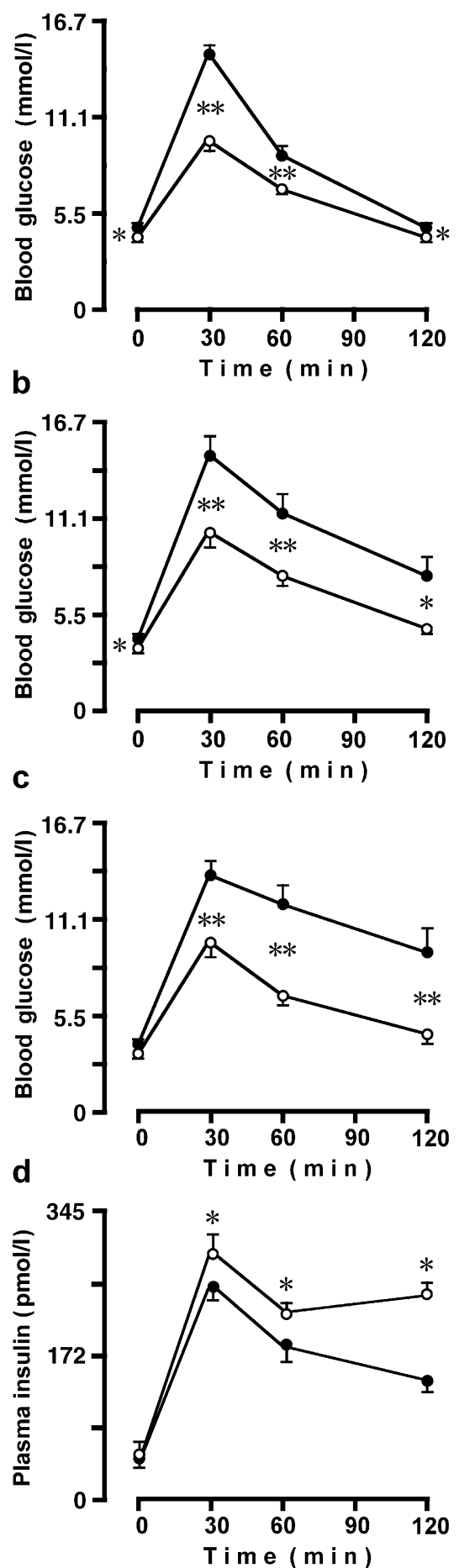

Fig. 6. Glucose levels and insulin secretion during the ipGTT. After $16 \mathrm{~h}$ of fasting, $2 \mathrm{mg} / \mathrm{g}$ body weight of glucose in physiological saline was intraperitoneally injected. Blood glucose concentrations were examined at $0,30,60$ and $120 \mathrm{~min}$ after glucose injection at 5 (a), 15 (b) and 25 (c) weeks. d. Plasma insulin concentrations were measured at the same time points. $* p<0.05$ vs littermates; $* * p<0.01$ vs littermates. Empty circles, CDK4 R24C-Tg mice; filled circles, littermates

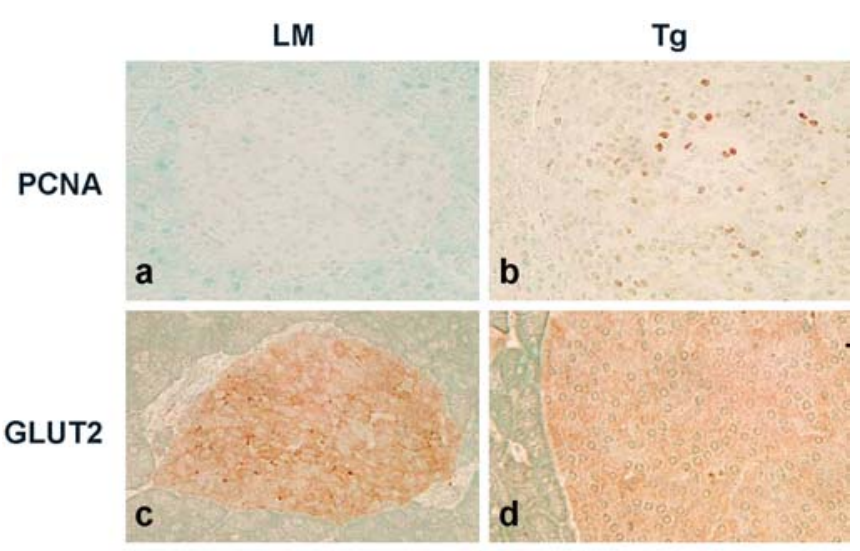

e

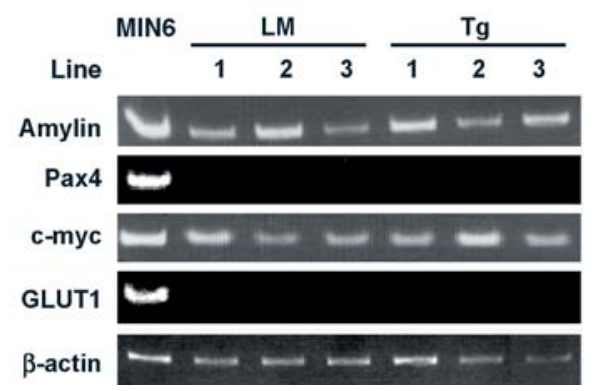

Fig. 7. Analysis of gene expression in pancreatic islets. Pancreata from littermates (a, c) and CDK4 R24C-Tg mice (b, d) at 25 weeks of age were examined by immunohistochemistry for PCNA $(\mathbf{a}, \mathbf{b})$ or GLUT2 $(\mathbf{c}, \mathbf{d})$. e. Islets obtained from five mice in the same line aged 20 to 25 weeks were used together for RNA extraction. Total RNA was subjected to semi-quantitative RT-PCR with specific primers for the indicated genes and for $\beta$-actin (internal control). MIN6 cells were used as a representative insulinoma cell line. LM, littermates; Tg, CDK4 R24C-Tg mice

dominant negative p53 permitted cells to divide beyond their normal limits [27]. In our system, the p53 pathway would suppress tumour formation by inhibiting the increase in telomerase activity and promoting the apoptosis of de-differentiated beta cells.

The results of the ipGTT confirmed that the beta cells in CDK4 R24C-Tg mice were highly differentiated. Although the early morning fasting blood glucose concentrations of CDK4 R24C-Tg mice were slightly lower than those of their littermates, they were within the normal range and were not hypoglycaemic. Fasting plasma insulin concentrations were similar in the two groups of mice. One of the diagnostic criteria for insulinoma is the presence of increasing plasma insulin concentrations even at fasting [28]. This criterion was not satisfied in CDK4 R24C-Tg mice. Furthermore, in line with the normal glucose concentrations observed in CDK4 R24C-Tg mice, insulin secretion was adequate and prompt in these mice after glucose loading. It has previously been shown that the precise regulation of insulin secretion is defective in the majority of patients with insulinoma 


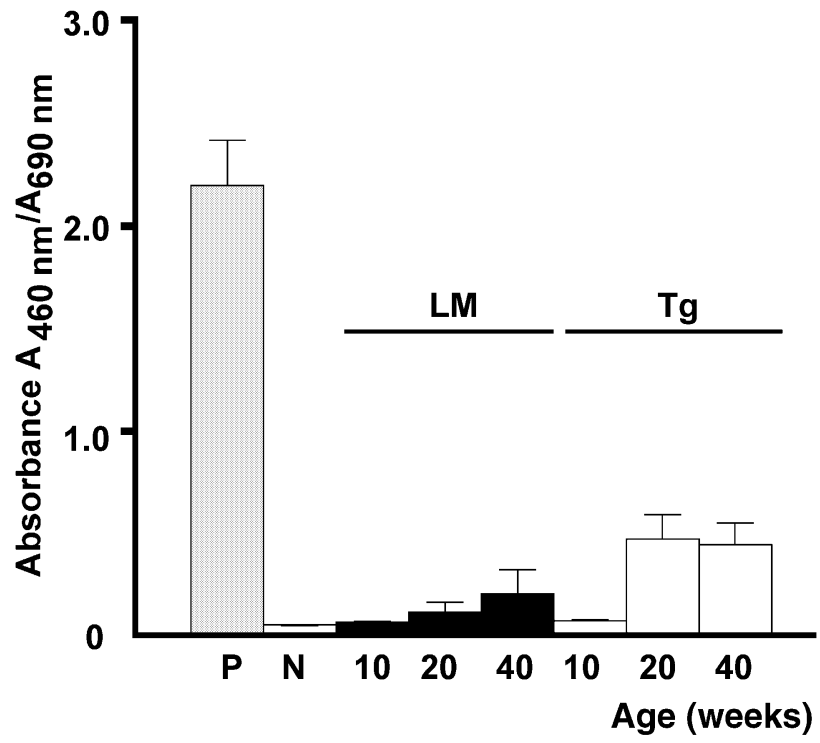

Fig. 8. Telomerase activites in pancreatic islets. Pancreatic islets were lysed in ice-cold lysis buffer and $10 \mu \mathrm{g}$ of protein extract was subjected to the telomerase PCR ELISA assay $(n=10$ each). Absorbance was measured at $450 \mathrm{~nm}$, with $690 \mathrm{~nm}$ used as a reference. MIN6 cell extract $(\mathrm{P})$ and lysis buffer $(\mathrm{N})$ were analysed as controls. LM, littermates; Tg, CDK4 R24C-Tg mice

[28]. Based on these observations, we concluded that insulinoma did not develop in the CDK4 R24C-Tg mice.

At 60 and 120 min after the intraperitoneal glucose injection, blood glucose concentrations increased with age in littermates. Because of an abundant food supply and hypomotility in the narrow cage, agedependent obese mice acquired insulin resistance. Although a similar increase in body weight was observed in CDK4 R24C-Tg, their blood glucose concentrations did not increase at 60 and 120 min with age. The CDK4 R24C-Tg mice possess the ability to secrete an adequate amount of insulin in response to increased insulin demand. At 25 weeks of age, the plasma insulin concentration curve of littermates after glucose injection showed one peak at $30 \mathrm{~min}$, and the concentration of insulin at $120 \mathrm{~min}$ was not high enough to suppress the blood glucose concentrations. Conversely, the insulin curve of CDK4 R24C-Tg mice showed two peaks, and a sufficient amount of insulin was secreted to appropriately control the blood glucose concentrations for $120 \mathrm{~min}$.

Although CDK4 R24C-Tg mice demonstrated the better glucose tolerance, $\mathrm{HbA}_{1} \mathrm{c}$ levels were not statistically different between CDK4 R24C-Tg mice and their littermates. This finding ruled out the possibility of chronic hypoglycaemia due to the constitutive excessive insulin secretion in CDK4 R24C-Tg mice. The differences in the capacity for insulin secretion could only be detected when both mice were intraperitoneally injected with $2 \mathrm{mg} / \mathrm{g}$ body weight of glucose. Blood glucose homeostasis is highly differentiated in CDK4
R24C-Tg mice such that dispensable insulin is not secreted except when glucose is loaded and a large amount of insulin is needed. It has recently been reported that levels of preproinsulin I and II mRNAs are not significantly increased in CDK4R24C/R24C mice, whereas there is a dramatic reduction in preproinsulin levels in $\mathrm{CDK}^{-/-}$mice [29]. These results suggest that CDK4 may play a critical role not only in postnatal pancreatic development, but also in the functional maturation of insulin secretion.

Even after 18 months, no insulinoma developed in the CDK4 R24C-Tg mice. Conversely, homozygous CDK4R24C/R24C knock-in mice have been shown to develop multiple tumours, including endocrine tumours and haemangiosarcomas, at the age of 1 year [6]. Heterozygous CDK4WT/R24C knock-in mice have also been observed to develop multiple tumours [8]. Given the powerful activity of the insulin promoter and the many copies of the CDK4 R24C transgene inserted into the genome of the CDK4 R24C-Tg mice, it is unlikely that the level of transgene expression in the CDK4 R24C$\mathrm{Tg}$ mice used in this study was lower than that in the knock-in mice used in previous studies. The level of CDK4 R24C expression driven by the insulin promoter in CDK4 R24C-Tg mice was four times higher than the level of CDK4 expression driven by the CDK4 promoter. These data rule out the existence of a threshold of CDK4 activity for tumourigenesis and indicate that tumour development is not determined simply by the level of CDK4 R24C expression.

Studies on transgenic mice expressing CDK4 in epidermis or astrocytes have reported that CDK4 overexpression alone is not sufficient to cause tumour formation $[30,31]$. The D-type cyclins control cyclin D/CDK4 activity by binding to and activating CDK4 [1]. However, it has been demonstrated that the overexpression of both cyclin D1 and CDK4 does not have a combined effect on tumour development $[32,33]$. CDK4 may have other cyclin partners and additional roles to the phosphorylation of $\mathrm{Rb}$. It is possible that CDK4 is regulated differently to CDK4 R24C since the amplification and overexpression of CDK4 has been observed in a wide spectrum of human tumours [34], whereas CDK4 R24C has been shown to be overexpressed only in sporadic and familial melanomas $[9,10]$.

The presence and the absence of neoplasms in CDK42 24C/R24C knock-in mice and CDK4 R24C-Tg mice respectively, suggests that the onset of CDK4 R24C expression may well govern neoplasm development. In knock-in mice with the germ line mutation, CDK4 R24C was expressed even in poorly differentiated beta cells, which could have caused beta cell transformation. The lack of malignant conversion in CDR4 R24C-Tg mice may be due to the fact that CDK4 R24C was only expressed in terminally differentiated beta cells under the control of the insulin promoter. 
CDK4 R24C-Tg mice in an N4 backcross to C57BL/6 or DBA2 showed a significant increase in islet area compared with their littermates, and this was similar to that observed in the original CDK4 R24C$\mathrm{Tg}$ mice in BDF1 background. The poor glucose tolerance of C57BL/6 mice compared with DBA2 wildtype mice was shown to be due to the genetic background of these littermates. In CDK4 R24C-Tg mice in an $\mathrm{N} 4$ backcross to C57BL/6 or DBA2, glucose regulation was intact and no hypoglycaemia was observed. These results indicate that the increase in islet area and the highly differentiated beta cell phenotype induced by CDK4 R24C overexpression are independent of the genetic background.

The proliferation and differentiation of beta cells are not induced concurrently, and the ability of highly differentiated beta cells to proliferate is thought to be extremely restricted. Using a genetic-lineage-marking approach, it has previously been shown that the maintenance of adult pancreatic beta cell mass in mice is not dependent on the stem cells, but on the self-duplication of pre-existing beta cells [35]. Beta cell area is determined by the balance between the rate of neogenesis or proliferation and the rate of apoptosis [36]. Levels of beta cell proliferation as assessed by 5-bromo-2'-deoxyuridine (BrdU) incorporation were 2.5 times higher in the islets of CDK4 $4^{\mathrm{R} 24 \mathrm{C} / \mathrm{R} 24 \mathrm{C}}$ mice than in the islets of their littermates at the age of 10 days [7]. The remarkable proliferation of beta cells observed in CDK4 R24C-Tg mice in our study demonstrated that beta cells could proliferate even at 25 weeks of age (i.e. at the highly differentiated stage) when the insulin promoter is functioning.

In this paper we have demonstrated in our transgenic mouse model that pancreatic islet beta cells proliferate significantly, with the preservation of highly differentiated phenotypes, irrespective of the genetic background. Based on this evidence, the postnatal activation of CDK4 in pancreatic islets may potentially be used for the therapeutic stimulation of proliferation of differentiated pancreatic islet beta cells. Human pancreatic islets transduced with CDK4 R24C by lentivirus show proliferative potential in response to glucose stimulation in vitro [37]. The postnatal activation of CDK4 through the use of new techniques (including the administration of vectors expressing $C D K 4$ $R 24 C$ under the control of the insulin promoter or CDK4-activating drugs) could potentially lead to the postnatal proliferation of pancreatic islet beta cells without neoplasm formation, thus providing a radical new treatment for diabetes.

Acknowledgements. This study was supported by a Grant-inAid for Scientific Research from Japanese Society for the Promotion of Science and a Cooperative Link of Unique Science and Technology for Economic Revitalization (CLUSTER). The authors are not aware of any conflicts of interest.

\section{References}

1. Sherr CJ (1996) Cancer cell cycle. Science 274:1672-1677

2. Kitagawa M, Higashi H, Jung HK et al. (1996) The consensus motif for phosphorylation by cyclin D1-Cdk4 is different from that for phosphorylation by cyclin A/E-Cdk2. EMBO J 15:7060-7069

3. Sherr CJ, Roberts JM (1999) CDK inhibitors: positive and negative regulators of $\mathrm{G}_{1}$-phase progression. Genes Dev 13:1501-1512

4. Yamaoka T, Itakura M (1999) Development of pancreatic islets. Int J Mol Med 3:247-261

5. Tsutsui T, Hesabi B, Moons DS et al. (1999) Targeted disruption of CDK4 delays cell entry with enhanced p27Kip1 activity. Mol Cell Biol 10:7011-7019

6. Rane SG, Dubus P, Mettus RV et al. (1999) Loss of CDK4 expression causes insulin-deficient diabetes and Cdk4 activation results in beta-islet cell hyperplasia. Nat Genet 22:44-52

7. Martin J, Hunt SL, Dudus P et al. (2003) Genetic rescue of Cdk4 null mice restores pancreatic beta-cell proliferation but not homeostatic cell number. Oncogene 22:5261-5269

8. Sotillo R, Dubus P, Martin J et al. (2001) Wide spectrum of tumors in knock-in mice carrying a Cdk4 protein insensitive to INK4 inhibitors. EMBO J 20:6637-6647

9. Wolfel T, Hauer M, Schneider J et al. (1995) A p16 ${ }^{\mathrm{INK} 4 \mathrm{a}_{-}}$ insensitive CDK4 mutant targeted by cytolytic T lymphocytes in a human melanoma. Science 269:1281-1284

10. Zou L, Weger J, Yang Q et al. (1996) Germline mutations in the p16 INK4a binding domain of CDK4 in familial melanoma. Nat Genet 12:97-99

11. Yamaoka T, Idehara C, Yano M et al. (1998) Hypoplasia of pancreatic islets in transgenic mice expressing activin receptor mutants. J Clin Invest 102:294-301

12. Hogan B, Beddington R, Costantini F, Lacy E (1994) Manipulating the mouse embryo. A laboratory manual, 2nd edition. Cold Spring Harbor Laboratory Press, New York

13. Moritani M, Yoshimoto K, Ii S et al. (1996) Prevention of adoptively transferred diabetes in nonobese diabetic mice with IL-10-transduced islet-specific Th1 lymphocytes. J Clin Invest 98:1851-1859

14. Gotoh M, Maki T, Kiyoizumi T, Satomo S, Monaco AP (1985) An improved method for isolation of mouse pancreatic islets. Transplantation 40:437-438

15. Kato A, Takahashi H, Takahashi Y, Matsushime H (1997) Inactivation of the cyclin D-dependent kinase in the rat fibroblast cell line, $3 \mathrm{Y} 1$, induced by contact inhibition. J Biol Chem 272:8065-8070

16. Matsushime H, Ewen ME, Strom DK et al. (1992) Identification and properties of an atypical catalytic subunit (p34PSK-J3/cdk4) for mammalian D type G1 cyclins. Cell 71:323-334

17. Yamada T, Brunstedt J, Solomon T (1983) Chronic effects of caerulein and secretion on endocrine of the rat. Am J Physiol 24:G541-G545

18. Kim NW, Piatyszek MA, Prowse KR et al. (1994) Specific association of human telomerase activity with immortal cells and cancer. Science 266:2011-2015

19. Asplund K (1973) Dynamics of insulin release from the fetal and neonatal rat pancreas. Eur J Clin Invest 3:338-344

20. Hole RL, Pian-Smith MC, Sharp GW (1988) Development of the biphasic response to glucose in fetal and neonatal rat pancreas. Am J Physiol 254:E167-E174

21. Otonkoski T, Anderson S, Knip M, Simell O (1988) Maturation of insulin response to glucose during human fetal and neonatal development. Diabetes 37:286-291 
22. Weinhaus AF, Poronnik P, Cook DI, Tuch BE (1995) Insulin secretagogues, but not glucose, stimulate an increase in $[\mathrm{Ca}++]$ in the fetal rat beta cell. Diabetes 44:118-124

23. Boden G, Murer E, Mozzoli M (1994) Glucose transporter proteins in human insulinoma. Ann Intern Med 121:109-112

24. Miyamoto T, Kakizawa T, Ichikawa K, Nishio, S, Kajikawa S, Hashizume K (2001) Expression of dominant negative form of PAX4 in human insulinoma. Biochem Biophys Res Commun 282:34-40

25. Pavelic K, Hrascan R, Kapitanovic S et al. (1995) Multiple genetic alterations in malignant metastatic insulinomas. J Pathol 177:395-400

26. Pavelic K, Hrascan R, Kapitanovic S et al. (1996) Molecular genetics of malignant insulinoma. Anticancer Res 16:1707-1717

27. Rheinwald JG, Hahn WC, Ramsey MR et al. (2002) A two-stage, p16 ${ }^{\mathrm{INK} 4 \mathrm{a}_{-}}$and $\mathrm{p} 53$-dependent keratinocyte senescence mechanism that limits replicative potential independent of telomere status. Mol Cell Biol 22:5157-5172

28. Fowler DL, Wood WG, Koontz PG Jr (1980) Endogenous hyperinsulinism. Am J Gastroenterol 74:321-327

29. Mettus RV, Rane SG (2003) Characterization of the abnormal pancreatic development, reduced growth and infertility in Cdk4 mutant mice. Oncogene 22:8413-8421

30. Miliani de Marval PL, Gimenez-Conti IB, LaCave M, Martinez LA, Conti CJ, Rodriguez-Puebla ML (2001) Transgenic expression of cyclin-dependent kinase 4 results in epidermal hyperplasia, hypertrophy, and severe dermal fibrosis. Am J Pathol 159:369-379

31. Huang ZY, Baldwin RL, Hedrick NM, Gutmann DH (2002) Astrocyte-specific expression of CDK4 is not sufficient for tumor formation, but cooperates with p53 heterozygosity to provide a growth advantage for astrocytes in vivo. Oncogene 21:1325-1334

32. Miliani de Marval PL, Macias E, Conti CJ, RodriguezPuebla ML (2004) Enhanced malignant tumorigenesis in Cdk4 transgenic mice. Oncogene 23:1863-1873

33. Lazarov M, Kubo Y, Cai T et al. (2002) CDK4 coexpression with Ras generates malignant human epidermal tumorigenesis. Nat Med 8:1105-1114

34. Ortega S, Malumbres M, Barbacid M (2002) Cyclin Ddependent kinases, INK4 inhibitors and cancer. Biochim Biophys Acta 1602:73-87

35. Dor Y, Brown J, Martinez OI, Melton DA (2004) Adult pancreatic beta-cells are formed by self-duplication rather than stem-cell differentiation. Nature 429:41-46

36. Bonner-Weir S (2000) Life and death of the pancreatic beta cells. Trends Endocrinol Metab 11:375-78

37. Marzo N, Mora C, Fabregat ME et al. (2004) Pancreatic islets from cyclin-dependent kinase 4/R24C (Cdk4) knockin mice have significantly increased beta cell mass and are physiologically functional, indicating that $\mathrm{Cdk} 4$ is a potential target for pancreatic beta cell mass regeneration in Type 1 diabetes. Diabetologia 47:686-694 